\title{
Coupled piezoelectric-circuit FEA to study influence of a resistive load on power output of piezoelectric energy devices
}

\author{
Meiling $\mathrm{Zhu}^{1 *}$, Emma Worthington ${ }^{1}$ and James Njuguna ${ }^{2}$ \\ ${ }^{1}$ Department of Materials, ${ }^{2}$ Department of Sustainable Systems \\ Cranfield University, Bedfordshire, MK43 0AL UK \\ *E-mail address: m.zhu@ cranfield.ac.uk.
}

\begin{abstract}
This paper presents, for the first time, a coupled piezoelectric-circuit finite element model (CPC-FEM) to analyze the power output of vibration-based piezoelectric energy harvesting devices (EHDs) when connected to a resistive load. Special focus is given to the effect of the resistive load value on the vibrational amplitude of the piezoelectric EHDs, and thus on the current, voltage, and power generated by the EHDs, which are normally assumed to be independent of the resistive load in order to reduce the complexity of modelling and simulation. The CPC-FEM presented uses a cantilever with the sandwich structure and a seismic mass attached to the tip to study the following load characteristics of the EHD as a result of changing the load resistor value: (1) the electric outputs of the EHD: current and voltage, (2) the power dissipated by the resistive load, (3) the vibration amplitude of tip displacement, and (4) the shift in resonant frequency of the cantilever. Significant dependences of the characteristics of the piezoelectric EHDs on the externally connected resistive load are found, rather than independency, as previously assumed in most literature. The CPC-FEM is capable of predicting the generated power output with different resistive load values while simultaneously considering the effect of the resistor value on the vibration amplitude. The CPC-FEM is invaluable for validating the performance of a device before fabrication and testing, thereby reducing the recurring costs associated with repeat fabrication and trials, and also for optimizing device design for maximal power-output generation.
\end{abstract}

Keywords: finite element method (FEM), vibration-based piezoelectric energy harvesting devices (EHDs), coupled piezoelectric-circuit (CPC), load characteristics.

\section{INTRODUCTION}

With technology advancements over the last few decades, the vast reduction in size and power consumption of complementary metal-oxide-semiconductor (CMOS) circuitry has led to a great research effort toward energy harvesting devices (EHDs) for the development of wireless sensors and ubiquitous wireless networks of communication nodes ${ }^{[1-5]}$. Significant progress has been made and a large number of vibration-based EHDs have been proposed and

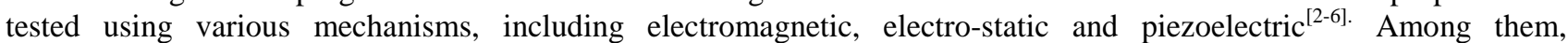
piezoelectric EHDs have received more attention due to their self-contained power without requiring external voltage source, highest energy density and good dynamic responses. The piezoelectric materials have the nature property of piezoelectric materials in highest electromechanical coupling between structural and electric fields, that is, applying a voltage to a piezoelectric material creates a displacement and/or vibrating a piezoelectric material generates a voltage. The natural property of piezoelectric materials has been widely used to transfer mechanical energy into electrical energy or vice verse. Thanks to the high electromechanical coupling coefficient of piezoelectric materials, EHDs made of piezoelectric materials are presently capable of scavenging energy in the range of $1-200 \mu \mathrm{W} / \mathrm{cm}^{3}$ from ambient vibration energy sources ${ }^{[5,7]}$ (possibly higher in the nearby future once optimized design and better piezoelectric materials are available). This energy level is suitable for power demand in the range of an average consumption of $100 \mu \mathrm{W}^{[8]}$, hence particularly suitable for wireless sensors and communication nodes. Cantilever-based piezoelectric EHDs with a seismic mass and sandwich structure are an attractive geometry for harvesting energy from vibration. The main reason for this is 
that such a structure is designed to work in the first bending mode with a lower stiffness, offering a lower resonant frequency, and easily designed to match the ambient vibration frequency for generating maximum power output.

The power output of EHDs dictates device suitability for most applications. As such, the evaluation of the power output of an EHD for a given excitation frequency and a given amplitude of ambient vibration plays an important role in the design of EHDs. Key to this is availability of a proper model that can be used to calculate the power output for designs.

According to modal analysis theory, the governing equations of piezoelectric EHDs can be written as follows:

$$
\begin{aligned}
& M \ddot{z}(t)+C \dot{z}(t)+K z(t)=M \ddot{y}(t)-\Theta V_{p} \\
& \Theta z(t)-C_{p} V_{p}(t)=Q_{p}(t)
\end{aligned}
$$

where: $M, C$ and $K$ represent the effective mechanical mass, mechanical damping and mechanical stiffness respectively; $\Theta$ represents the effective electromechanical coupling coefficient of a piezoelectric structure; $V_{p}$ and $Q_{p}$ represent the voltage across the piezoelectric electrodes and the electric charge on the electrodes respectively; $z(t)$ is the mechanical displacement of mass relative to the fixed point of the cantilever; $\ddot{y}(t)$ is the acceleration applied at the fixed point of the device from the ambient vibration environment; and $C_{p}$ is the electric capacitance of a piezoelectric structure.

Piezoelectric EHDs are often connected with an electric circuit to convert the harvested electric energy into a usable form $^{[9-12]}$. The circuit may include: a rectifier to convert AC to DC, a super-capacitor to store harvested energy, an inductive load to boosts the voltage output of the transducer, or a resistive load, used to characterize the capability of the designed EHD. Most existing modelling techniques assume the vibration amplitude of the EHD device to be independent of the connected circuit, however, the EHD output voltage and current are greatly influenced by the circuit connected. The question to answer is: how does the connected circuit influence the vibration response, and in turn the power output, of a piezoelectric EHD? At the present state of knowledge, this is unanswered in the literature for EHDs and attached circuits. This paper studies the effect of the value of that load on the power output of piezoelectric EHDs.

General equations for the electric outputs of a piezoelectric EHD directly connected with a load resistor are

$$
\begin{aligned}
& V_{R}(t)=I_{R}(t) R \\
& P_{R}=V_{R} I_{R}
\end{aligned}
$$

where: $V_{R}$ represents the voltage across the resistive load; and $I_{R}$ the current through it; $P_{R}$ represents the power dissipated in the resistive load, which is a good indication of the actual power generated by the piezoelectric EHD.

For the configuration where a piezoelectric EHD is directly connected with a load resistor, the relationships between the output of the piezoelectric EHD and the input to the resistive load are

$$
\begin{aligned}
& V_{p}(t)=V_{R}(t) \\
& I_{R}(t)=\omega Q_{p}(t)
\end{aligned}
$$

From (1)-(6), it is observed that $z(t), V_{R}(t)$ and $I_{R}(t)$ (and therefore $P_{R}(t)$ ) are coupled together through $R$ and $\Theta$. This means that $z(t), V_{R}(t)$ and $I_{R}(t)$ are all affected by $R$ and $\Theta$.

The present paper proposes a coupled piezoelectric-circuit finite element model (CPC-FEM) to study the power output, particularly focusing on the influence of the load resistor value, on the vibration amplitude (mechanical displacement) of the beam, therefore electric outputs of current, voltage, and power. The effects of electromechanical coupling on the vibration and electric outputs current, voltage and power are all simulated using the developed CPC-FEM. The motivation of this paper is to develop an understanding of how an externally connected electric load affects the power related output. Firstly, existing modelling techniques are briefly reviewed. A coupled piezoelectric-circuit FEM are then developed, in which piezoelectric EHDs are directly connected with an external electric circuit to calculate the electric outputs (current, voltage and power), the tip displacement, and the frequency shift. This is the first time in the literature CPC-FEM has been developed to perform such calculations. 


\section{AN BRIEF REVIEW ON EXISTING MODELLING METHODS}

A variety of modelling approaches have been used to analyse the output of EHDs. Among them, uncoupled analyses, equivalent electric circuit methods, and advanced modelling methods have been proposed. An overview of these existing methods is given in this section and the justification for the proposed CPC-FEM is explained.

\subsection{Uncoupled analyses}

Piezoelectric EHDs are complicated electromechanical dynamic systems. For simplicity, such devices have frequently been modelled as a current source in parallel with the piezoelectric capacitance $C_{p}{ }^{[7-13]}$. The assumption made is that the vibration amplitude of the piezoelectric structure is independent of the impedance of the externally connected load. This means that the externally connected load has no influence on the amplitude of the vibration displacement. Reverting to the governing equations ( 1 and 2 ) of piezoelectric EHDs, it can be easily seen that this assumption has ignored the coupling term $\Theta V_{R}$ on the right hand side of (1), reducing it to (7)

$$
M \ddot{z}(t)+C \dot{z}(t)+K z(t)=M \ddot{y}(t)
$$

Equation (7) represents an uncoupled system between electric and mechanical responses. This assumption is fairly reasonable in the case of extremely low coupling for sensor analyses. However, piezoelectric EHDs use a high electromechanical coupling material to transform mechanical energy to electric energy and so they are an exceptional case, and the assumption is not valid. This is because the generated electric energy in turn influences mechanical vibrations through the high electromechanical coupling term, $\Theta V_{R}$ on the right hand side of (1). This means that the harvested electric energy (voltage across/current fed into the resistor) from the ambient vibration will reduce the structural vibration, and this in turn reduces the harvested energy due to the reduced structural vibration. The evaluation of these coupling effects on the output capability of a piezoelectric EHD is an important task that requires attention. This paper proposes CPC-FEM to study this.

\subsection{Equivalent electric circuit methods}

Stand alone piezoelectric sensors without connection to an external circuit are normally modelled as a short circuit where current source is in parallel with the piezoelectric capacitance $C_{p}$, or as an open circuit where voltage source is in series with $C_{p}$. The modelling method is based on the following piezoelectric constitutive equations:

$$
\begin{aligned}
& \sigma_{i}=c_{i j} \varepsilon_{i}-e_{3 i} E_{3} \\
& D_{3}=e_{3 i} \varepsilon_{i}+\varepsilon_{3}^{\varepsilon} E_{3}
\end{aligned}
$$

In these equations, $\sigma_{i}, \varepsilon_{i}$, and $c_{i j}$ denote in turn components of the stress and strain vectors and components of stiffness matrices measured at constant electrical fields; $E_{3}$ and $D_{3}$ are the $z$-components of the electric field and displacement; $e_{3 i}$ the piezoelectric stress coefficients measured at constant strains; and $\varepsilon_{3}^{\varepsilon}$ the $z$-component of dielectric coefficient measured at constant strains. For the calculation of the current source as a short circuit for sensing analyses, it is assumed that $\varepsilon_{i}$ is fixed, and let $E_{3}=0$ in (9). One can then calculate the electric charge generated in the electrode surfaces and the electric current flowing through the electrodes based on $D_{3}=e_{3 i} \varepsilon_{i}$. The vibration is calculated based on $\sigma_{i}=c_{i j} \varepsilon_{i}$ as the case considered is short circuit and so $E_{3}=0$. For the calculation of a voltage source as an open circuit, a similar approach as the short circuit is used. That is, assuming $\varepsilon_{i}$ is fixed and letting $D_{3}=0$. In (9) one can then calculate the electric voltage across piezoelectric electrodes based on $E_{3}=-e_{3 i} \varepsilon_{i} / \varepsilon_{3}{ }^{\varepsilon}$. The above calculations are valid for charge or voltage calculations only as the electric conditional circuits are in the open or short circuit conditions.

For piezoelectric EHDs connected with external electric circuits, using the above equivalent electric circuit theory to calculate currents or voltages of the devices is not very well suited, as the EHD is not in an open or short-circuit condition. Calculation of currents and voltages by using these methods would over-estimate, also leading to an overestimation in power. 


\subsection{Advanced modelling methods}

A few advanced models to analyze piezoelectric EHDs have also been reported in the literature. For example, Guyomar et al. ${ }^{[12]}$ proposed a new approach for the case of coupling not so weak to estimate the average harvested power. In their approach, the assumption made was that the external forcing function $M \ddot{y}(t)$ and the velocity of the mass $\dot{z}(t)$ are in phase. This assumption is fairly reasonable for non-piezoelectric mechanical structures operating around resonance with low damping. Whether this assumption still holds for the case of non-small electromechanical coupling is questionable and has not been addressed. Shu and Lien ${ }^{[7]}$ developed an analytical model of power output for piezoelectric harvesting systems focusing on AC-DC output. Their research concluded that the harvested power depends on the input vibration characteristics (frequency and acceleration, the mass of generator, the electric load, the natural frequency, the mechanical damping ratio and the electromechanical coupling coefficient of system). Further analytical modelling and analysis of micro piezoelectric power generators was reported by $\mathrm{Lu}$ who focused on the influence of load resistance on the output power of cantilevered piezoelectric bimorph ${ }^{[14]}$.

It is not difficult to find that all the advanced modelling and simulation mentioned above are reliant on the availability of parameters of $M, K, C$ and $\Theta$. These parameters vary in different structures; subtle changes in geometry and mass location will change the parameters. Derivation of each individually designed structure would be a very time-consuming task.

FEA is an advanced and reliable numerical modelling method, which has already been used to calculate sensors and actuators in numerous applications, and used to calculate open circuit and short circuit conditions for piezoelectric EHDs $^{[14]}$. However, FEA has not been used to predict the power output of piezoelectric EHDs that are connected to an external electric circuit, possibly because a suitable FEA model has not been put forward to date due to the complexity in analyses. This paper will present a CPC-FEM to study the effects of a resistive load on the vibration and also on the electric outputs: voltage, current and power, of the EHD.

\section{CPC-FEM MODELLING AND SIMULATION}

Commercially available FEA software (ANSYS Inc. Canonsburg, PA) ${ }^{[15]}$, was utilized to develop the CPC numerical model of the piezoelectric EHD connected directly with a resistive load. Special focus was given to the analysis of power related outputs and vibrational characteristics, where the 'power related outputs' represents the current through and voltage across the resistor and the power dissipated in it, and the 'vibrational characteristics' represents the tip displacement and the resonant frequency shift of the piezoelectric EHD.

\subsection{Configuration of Modelling}

The modelled geometry of a piezoelectric cantilever beam with a seismic mass is shown in Figure 1. The beam is a piezoelectric sandwich structure with a central brass substrate layer and two piezoelectric material layers, one each bonded to the top and bottom of the brass layer. The two piezoelectric layers can be wired in series so that their individual voltages add together to feed into an externally connected circuit (as shown in Figure 2), or in parallel so that their individual currents add together to feed into a connected resistor, as shown in Figure 3. In series, the two piezoelectric layers are oppositely polarized and the output terminals are the outside electrodes of the piezoelectric layers. In this case, an electric potential $\left(2 V_{p}\right)$ exists between the output terminals when the structure is subjected to a vibration at the fixed end of the beam. In parallel, the two piezoelectric layers are polarized in the same direction, and the outside electrodes are joined together to make one terminal. The central layer constitutes the other terminal. In this configuration, the same amount of charge exists on each outside electrode when the structure is subjected to vibration at the fixed end. These two configurations: parallel and series, were each studied for the comparison of the EHD outputs.

In developed finite element models (FEMs), the direction of polarization of the piezoelectric material is represented by the sign of the piezoelectric coefficients $\mathrm{e}_{31}$ and $\mathrm{e}_{33}{ }^{[15]}$. For example, in series, the polarization of the bottom piezoelectric layer is opposite to that of top piezoelectric layer, shown in Figure 2, so $\mathrm{e}_{31}$ and $\mathrm{e}_{33}$ have opposite signs in the defined material property to the top piezoelectric material in the developed model. The electrode connections have been made by using the 'couple' commands, creating two common nodes for the piezoelectric EHD to fulfil the required connection. The external connected circuit is then connected with these two common nodes in the model. The location of the circuit with respect to the device can be arbitrary as it does not affect the analytical results. As the adhesives between the substrate and piezoelectric layers are very thin, the adhesive thicknesses are ignored in all simulations. 


\subsection{CPC-FEM}

Figure 4 shows the developed CPC-FEM: a sandwich cantilever beam with a piezoelectric layer on the top and bottom, and a seismic mass attached to the tip, that is directed connected to a load resistor. The 8-node, hexahedral, coupled-field element SOLID5 is used for the piezoelectric material, and the 8-node, linear, structural element SOLID45 for the nonpiezoelectric material. To achieve this coupled piezoelectric-circuit analysis, the piezoelectric circuit element (CIRCU94) is connected with the piezoelectric element (SOLID5); (CIRCU94 can model resistors, inductors, capacitors, current sources, or voltage sources). In this model, CIRCU94 is used to model the load resistor to study the power related outputs and vibrational characteristics. The resistance value is defined by a real constant in the developed programme. The CIRCU94 resistor element is directly connected to the EHD through defined coupled common nodes, and the detailed connections are based on either the series or parallel configuration, shown in Figure 2 or 3.

The modelled geometric parameters and material properties are listed in Table 1, where they are purposely fixed to enable a focus of analyses on the effect of different load resistor values on the power related outputs and vibrational characteristics. The piezoelectric material chosen was PSI-5H4E from Piezo Systems, Inc. This material has a very high piezoelectric constant, therefore devices made with it are able to harvest higher amounts of energy from the ambient vibration environment. All the geometric dimensions are taken from Ref. ${ }^{[16]}$. The dielectric constant loss is ignored but the piezoelectric material loss is accounted for, which is based on the piezoelectric mechanical quality factor $Q$. This was introduced into the developed FEM model by use of the constant damping ratio $\xi_{\text {p }}$, which is the ratio between the actual damping and critical damping in the harmonic analysis, and is obtained from the relationship $\mathrm{Q}=\frac{1}{2 \xi_{p}}$ (the $Q$ value is 32 for the selected piezoelectric material). Elastic constants are obtained basing on the conversion of the elastic modulus and Poisson's ratio ${ }^{[17-19]}$. The substrate is made of brass and the seismic mass is made of tungsten-alloy for generating higher electric energy. For simulation, the input vibration has a constant vibrational amplitude of displacement of $3.96 \mu \mathrm{m}$, which acts at the fixed end of the cantilever, simulating an ambient vibration environment. The value of the resistive load is variable. Harmonic analyses are first performed with different resistive load. The electric current, voltage and power output of the EHD is extracted from the output data of SOLID5 and CIRCU94 to evaluate the relationships of $V_{p}(t)=V_{R}(t)$ and $I_{p}(t)=I_{R}(t)$, and also to confirm consistent with the calculation results. The tip-displacements and resonant frequencies were also extracted from the simulation results. The results are shown in Figures 5-11 and Tables 2.

\section{RESULTS AND DISCUSSIONS}

\subsection{Frequency responses of configuration in parallel}

Figure 5 shows the simulated frequency response for the current and voltage outputs of the EHD (piezoelectric layers in parallel configuration) with the following circuit connections: (1) short circuit where $R=10^{-20}$ in the developed CPCFEM as it is impossible to let $R=0$ perform the simulation; (2) open circuit where $R=10^{20}$, again, it is impossible to let $R=\infty$; (3) a circuit connected with a resistor $R_{o p t}$, where $R_{o p t}=\frac{1}{\omega_{n} C_{p}}\left(\omega_{n}\right.$ being the resonant frequency of the EHD). It can be seen from Figure 5 that for $R=0$, the piezoelectric EHD is a current source and $V_{R}=0$ (not shown in Figure 5 as $V_{R}=0$ ). All the current generated stays in the piezoelectric capacitor $C_{p}$, thus there is no electric current feeding into the output terminals and so the output power from the EHD is 0 , i.e., $P_{\text {out }}=0$. Similarly, for $R=\infty$, the piezoelectric EHD is a voltage source and $I_{P}=0$ (not shown in Figure 5 as $I_{R}=0$ ), the output power from the EHD is 0, as $I_{R}=I_{p}=0$, i.e., $P_{o u t}=0$. But, for $R_{o p t}=\frac{1}{\omega_{n} C_{p}}$, it can be seen that power is generated by the piezoelectric EHD, as there is current flowing through, and voltage developed across, the connected load resistor.

Figure 6 shows the simulated frequency response of the dissipated power and tip-displacement of the piezoelectric EHD for the same circuit connections as those for Figure 5. Here, the tip-displacement is greatly affected by the connected resistive load, as the amplitude at the resonant frequency decreases from $169 \mu \mathrm{m}$ to $71 \mu \mathrm{m}$ and then increases to $178 \mu \mathrm{m}$ for $R=0 \rightarrow R_{\text {OPT }} \rightarrow \infty$, it shifts from $78.5 \mathrm{~Hz}$ to $82 \mathrm{~Hz}$, and then to $83 \mathrm{~Hz}$ for $R=0 \rightarrow R_{O P T} \rightarrow \infty$, and the power output 
at the resonant frequency changes from $0 \mu \mathrm{W}$ to $60.6 \mu \mathrm{W}$ and then back to $0 \mu \mathrm{W}$ for $R=0 \rightarrow R_{\text {OPT }} \rightarrow \infty$. The connected resistive load has an obvious damping effect on the vibrational amplitudes, but it is not the case that the larger the resistive load, the higher the damping effect; it is more complex and difficult to explain than that. However, it can be concluded that the connected resistor has a significant effect on the vibrational amplitude of the piezoelectric EHD, and on the current, voltage and power in turn. Therefore, the coupling effect of the connected resistive load on the vibration characteristics needs to be considered for correct analysis of the piezoelectric EHD and its power output.

\subsection{Frequency responses of the configuration in series}

Similar conclusions for piezoelectric layers in series can be obtained as for those in parallel. Tables 2 summarize the characteristics of the EHDs of piezoelectric layers in parallel and in series for different circuit connections.

It is also interesting to find that the different configurations change the distribution of the current and voltage at the output terminals but do not significantly affect the overall power output and vibrational amplitude.

\subsection{Load characteristics}

To further evaluate the effect of the connected resistive load on the performance of the piezoelectric EHD, Figures 9, 10 and 11 show the currents and voltages, dissipated powers in the resistor, tip-displacements at the end of the beam, and the resonant frequency shift versus a range of electric resistance values for the parallel configuration. The current and voltage at maximum power output versus resistance value are indicated in Figure 9 and the corresponding vibrational amplitude and power are also indicated in Figure 10. Similar conclusions for the load characteristics can be obtained as for the simulated frequency response results, that is, (1) the current, voltage, and power dissipated depend significantly on the value of the load resistance, and (2) the value of the load resistance also significantly affects the vibrational characteristics of a piezoelectric EHD, including the vibrational amplitude and the resonant frequency. From further analysis of the simulation curves, some important results can be obtained: (1) the maximum power output of piezoelectric EHDs does not appear at the maximum vibrational displacement, because as has been proven, the power is determined by the product of current and voltage, and (2) the maximum power output does not appear at the $R_{o p t}$, as normally presented in literature. The reason for this is possibly that the model proposed here has taken into account the vibrational amplitude that is affected by the externally connected resistance while the literature has to date ignored these crucial effects.

\section{CONCLUSIONS}

A CPC-FEM is, for the first time, proposed to study the power output of a vibration-based piezoelectric EHD that is directly connected to a resistive load. The effect of the resistive load on the vibration amplitude of the EHD, and therefore on the current, voltage and power generated by the EHD, are investigated. These parameters are normally assumed to be independent of the resistor value for the reduction in complexity of modelling and simulation. The studied CPC-FEM of the piezoelectric EHD is based on a cantilever with a sandwich structure and a mass attached at the tip. The load characteristics, including (1) the electric outputs (current and voltage), (2) the power dissipated by the connected resistive load, (3) the tip vibration displacement amplitude, and (4) the resonant frequency shift of the cantilever versus a externally connected resistive load, are all simulated using the developed CPC-FEM. Significant dependences of the vibration characteristics of the piezoelectric EHDs on the externally connected resistive load are found, rather than independency. One interesting and important result found from the analyses is that the maximum power output of piezoelectric EHDs does not appear at the maximum vibrational displacement, as the power is determined by the product of electric current and electric voltage. The presented CPC-FEM has the ability to calculate the generated power output and responded vibration of vibration-based piezoelectric EHDs for a range of resistive loads and frequencies, and it has potential to be used for the optimization of piezoelectric EHD designs for the generation of maximal power output in a given volume.

In addition, the configurations of piezoelectric layers in parallel and in series have been studied. It is found that the configurations can change the distribution of the electric current and voltage at the output terminals but cannot change significantly the power output and vibrational amplitude when the structure is subjected to same excitations.

\section{ACKNOWLEDGEMENT}


The authors gratefully acknowledge the financial support from the Cranfield Innovative Manufacturing Research Centre via EPSRC entitled "Design and Manufacturing of a Novel EHDs for Self Powered Sensors and ICT Applications" (IMRC-137), and platform programme (EP/D506638/1) entitled "Nanoscale Multifunctional Ferroic Materials and Devices".

\section{References}

1. Roundy, S., Wright, P. K. and Rabaey, J., "A study of low level vibrations as a power source for wireless sensor nodes," Comput. Commun. 26(11), 1131-1144 (2003).

2. Mitchson, P. D., Yeatman, E. M., Rao, G. K. Holmes, A. S. and Green, T.C., "Energy harvesting from Human and machine motion for wireless electronic devices," Proc. IEEE 96(9), 1457-1486 (2008).

3. C. Kompis and S. Aliwell, "Energy harvesting technologies to enable romote and wireless sensing," Sensors and Instrumentation-Knowledge Transfer Network, June 2008.

4. Priya, S. "Advances in energy harvesting using low profile piezoelectric transducerds", J Electroceramics 19(1), 165-182 (2007).

5. Spies, P., Pollak, M. and Rohmer, G., "Power management for energy harvesting applications", available on line: http://www.iis.fraunhofer.de/Images/power_management_for_energy_harvesting_applications_tcm278-91133.pdf (Accessed $9^{\text {th }}$ Mar. 2009).

6. Beeby, S. P., Tudor, M. J. and White, N. M. "Energy harvesting vibration sources for microsystems applications," Measur. Sci. Technol. 17(12), R175-R195 (2006).

7. Shu, Y. C. and Lien, I. C., "Analysis of power output for piezoelectric energy harvesting systems," Smart Mater. Struct. 15(6) 1499-1512 (2006).

8. Roundy, S., Leland, E. S., Baker, J., Carleton, E., Reilly, E., Lai, E., Otis, B., Rabaey, J. M., Wright, P. K. and Sundararajan, V., "Improving power output for vibration-based energy scavengers," Pervasive Comput. IEEE 4(1), 28-36 (2005).

9. Lefeuvre, E., Badel, A., Richard, C. and Guyomar, D., "Piezoelectric energy harvesting devices optimization by synchronous electric charge extraction,” J. Intellig. Mater. Syst. Struct. 16(10), 865-876 ( 2005).

10. Lefeuvre, E., Badel, A., Richard, C., Petit, L. and Guyomar, D. "A comparison between several vibration-powered piezoelectric generators for standalone systems," Sensors and Actuators A 126(2), 405-416 (2006).

11. Lesieutre, G. A., Ottman, G. K. and Hofmann, H. F., "Damping as a result of piezoelectric energy harvesting," J. Sound Vibr. 269(3-5), 991-1001 (2004).

12. Guyomar, D., Badel, A., Lefeuvre, E. and Richard, C. "Toward energy harvesting using active materials and conversion improvement by nonlinear processing," Ultrasonics, Ferroelectr. Freq. Contr., IEEE Trans 52(4), 584$595(2005)$.

13. Ottman, G. K., Hofmann, H. F. and Lesieutre, G. A., "Optimized piezoelectric energy harvesting circuit using stepdown converter in discontinuous conduction mode," Power Electr., IEEE Trans 18(2), 696-703 (2003).

14. Lu, F., Lee, H. P. and Lim, S. P., "Modelling and analysis of micropiezoelectric power generators for microelectromechanical-systems applications," Smart Mater Struct 13(1), 57-63 (2004).

15. ANSYS, "ANSYS Release 11.0 Documentation", (2007)

16. Roundy, S., Wright P. K., and Rabaey, J. M., Energy Scavenging for Wireless Sensor Networks: With Special Focus on Vibrations," Kluwer Academic Publishers, Boston (2004).

17. Zhu, M., Kirby P. and Lim, M. Y., "Lagrange's formalism for modeling of a triaxial microaccelerometer with piezoelectric thin-film sensing," Sensors J, IEEE, 4(4), 455-463 (2004).

18. Zhu, M., and Leighton, G., "Dimensional reduction study of piezoelectric ceramics constitutive equations from 3-D to 2-D and 1-D," Ultrasonics, Ferroelectr. Freq. Contr., IEEE Trans 55(11), 2377-2383, (2008).

19. Whitney, J. M., "Structural Analysis of Laminated Anisotropic Plates." CRC Press, (1987),

20. Cross, C. J. and Fleeter, S., "Shunted pizoelectrics for passive control of turbomachine blading flow-induced vibration," Smart Mater. Struct. 11(2), 239-248 (2002).

21. Corr, L. R., and Clark, W. W., "Comparison of low-frequency piezoelectric switching shunt techniques for structural damping," Smart Mater. Struct. 11(3), 370-376 (2002). 


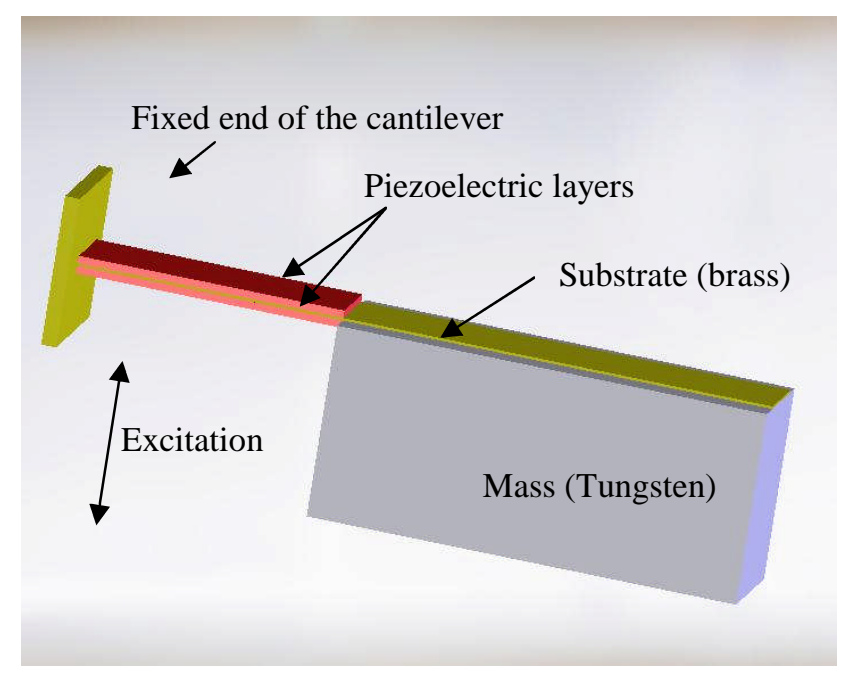

Figure 1 Modelled geometry of piezoelectric cantilever beam with a seismic mass

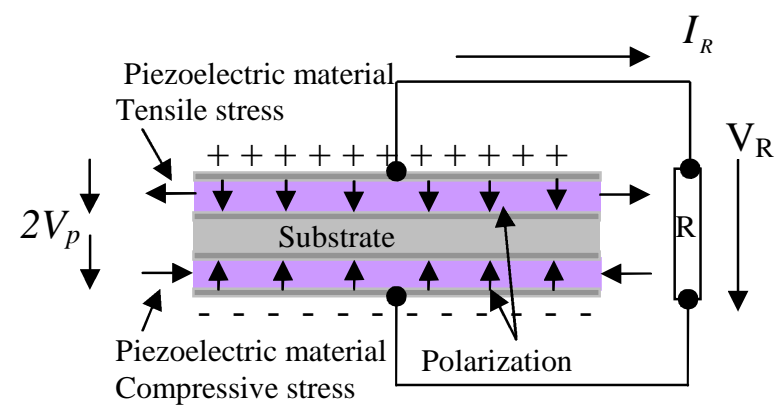

Figure 2 Configuration of piezoelectric layers in series, where "+” represents the plus charges and "-“ the minus charge

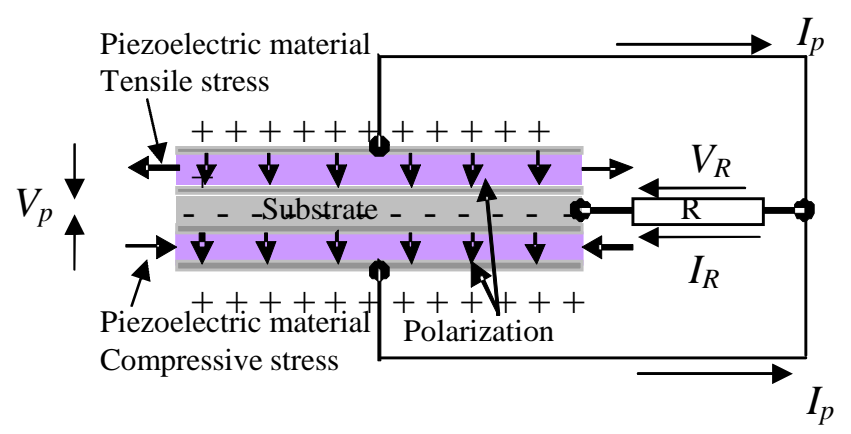

Figure 3 Configuration of piezoelectric layers in parallel, where "+” represents the plus charges and "-“ the minus charge 


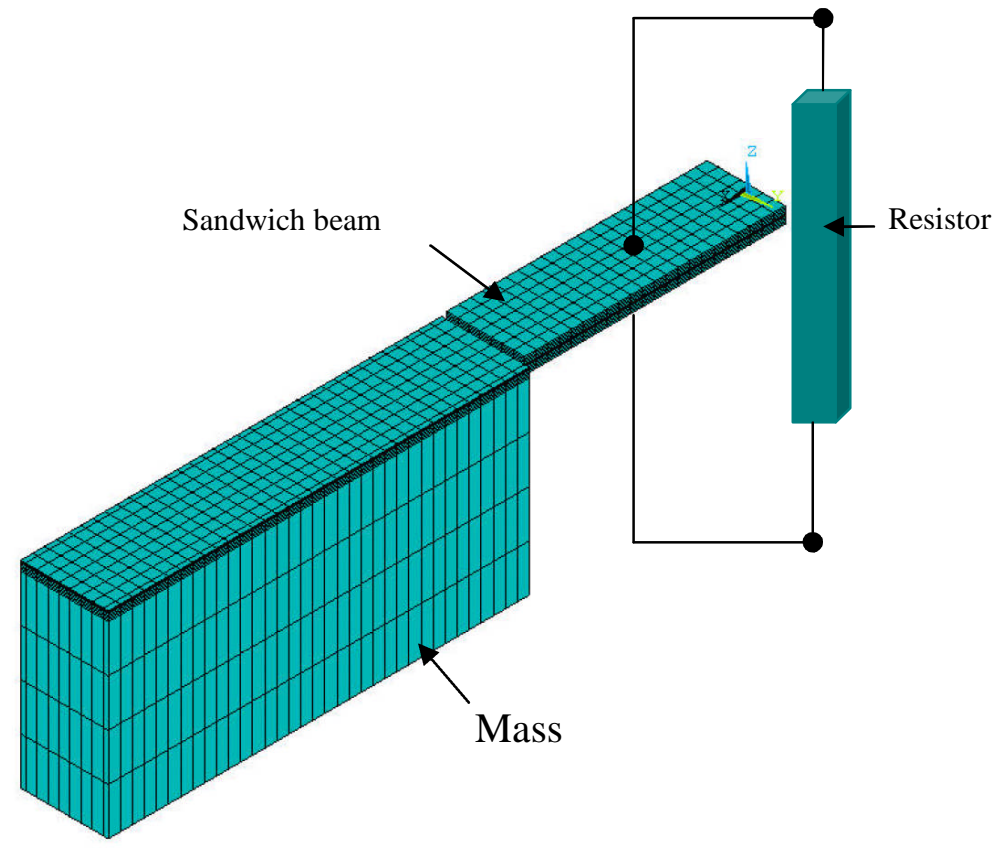

Figure 4 Developed CPC-FEM model of the cantilever beam of piezoelectric layers that are directly connected with a resistive load

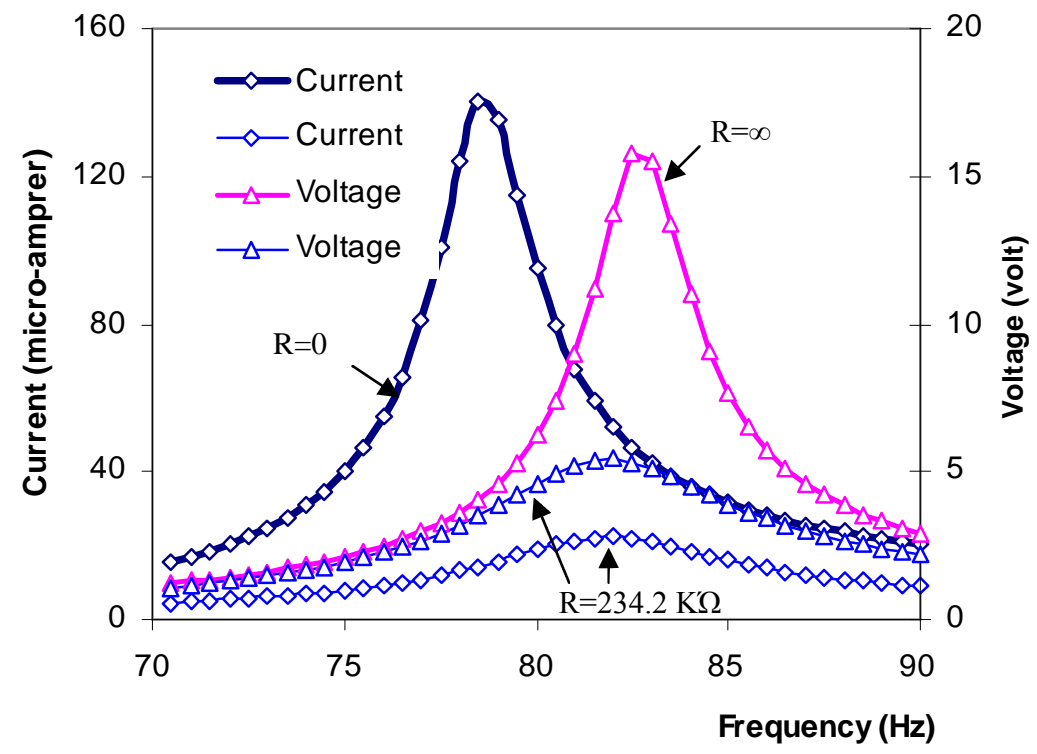

Figure 5 Simulated frequency responses for electric currents and electric voltages of piezoelectric EHDs of piezoelectric layers in parallel for the case of short circuit $(R=0)$, open circuit $(R=\infty)$, and circuit connected with a resistance $\left(\mathrm{R}=234.2 \mathrm{~K}^{\prime} \Omega\right)$ 


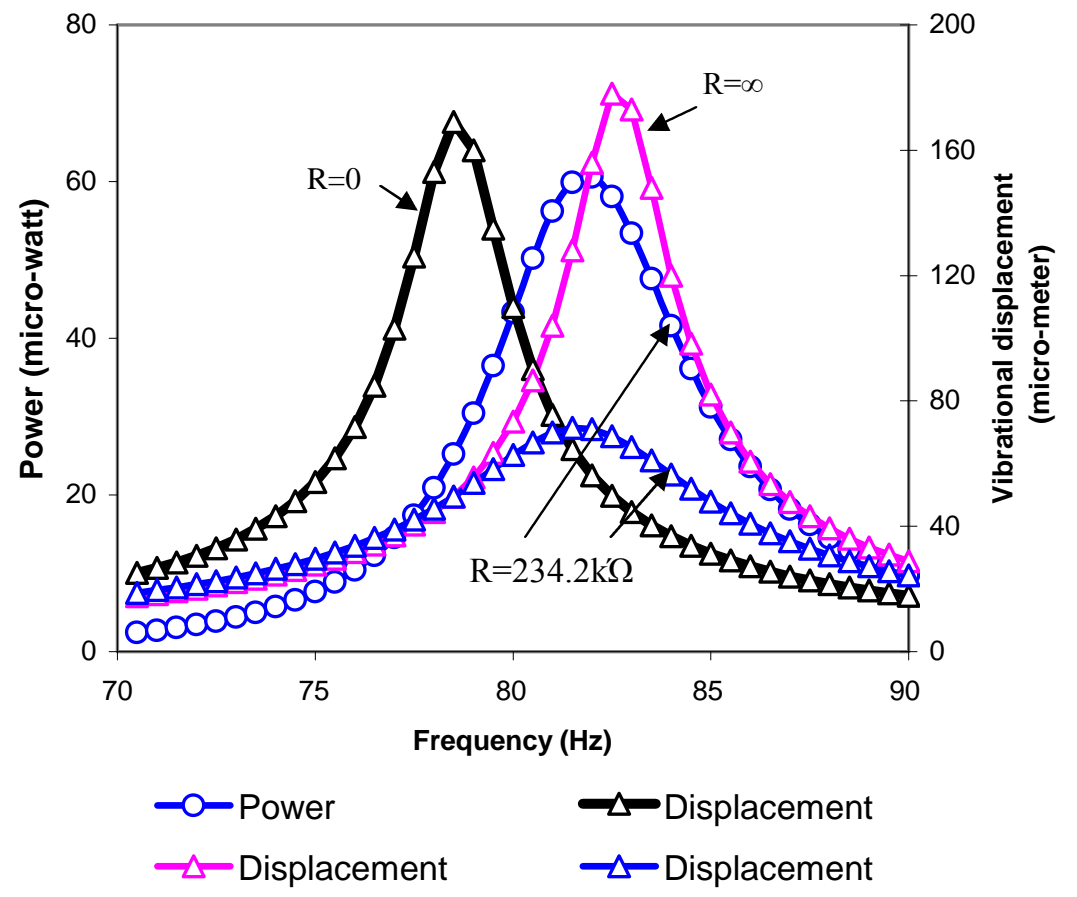

Figure 6 Simulated frequency responses for dissipated powers and tip vibration displacement amplitudes of piezoelectric EHDs of piezoelectric layers in parallel for the case of short circuit $(R=0)$, open circuit $(R=\infty)$, and circuit connected with a resistance $(R=234.2 \mathrm{~K} \Omega)$

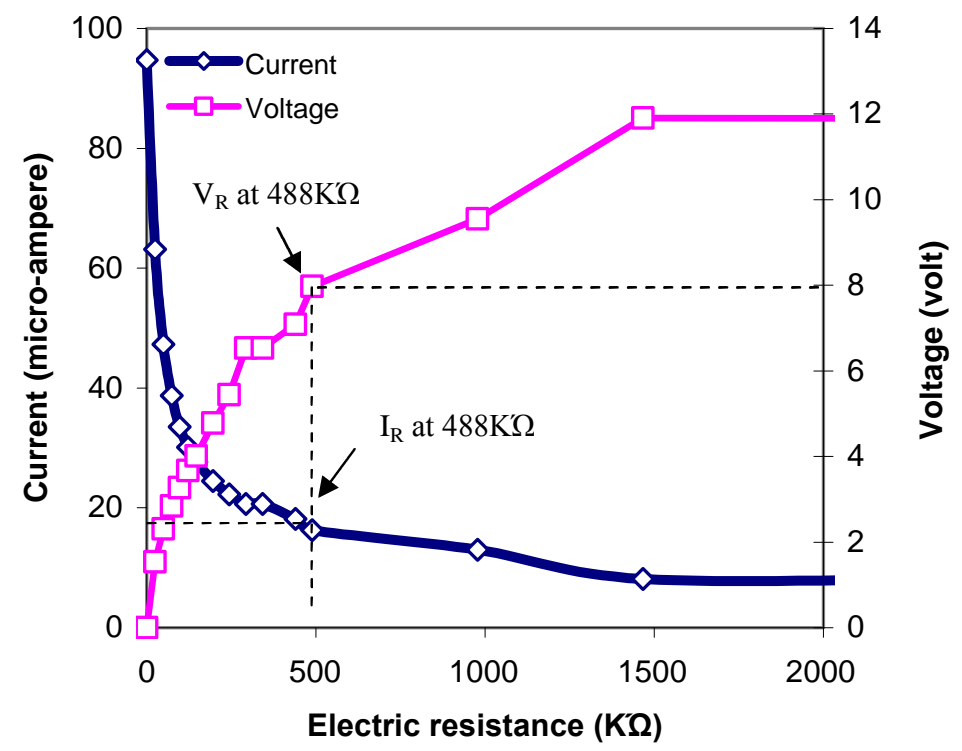

Figure 9 Electric currents fed into and voltages across a resistor verse the electric resistance connected to piezoelectric EHDs of piezoelectric layers in parallel 


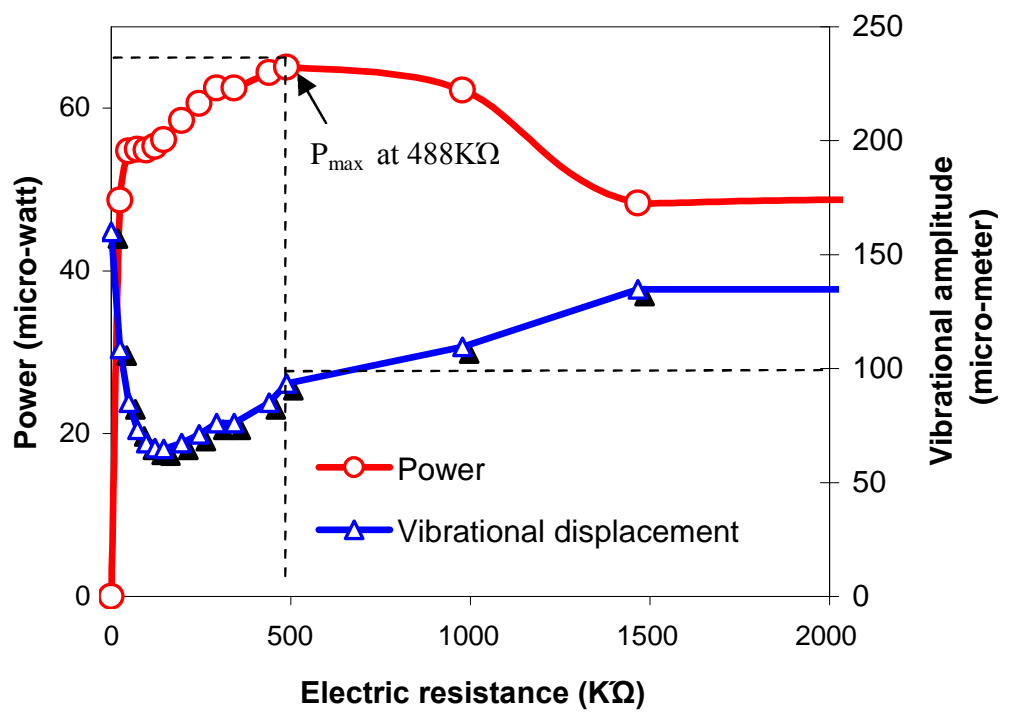

Figure 10 Dissipated powers by a resistor and tip vibrational displacement amplitudes of the beam verse electric resistances connected to piezoelectric EHDs of piezoelectric layers in parallel

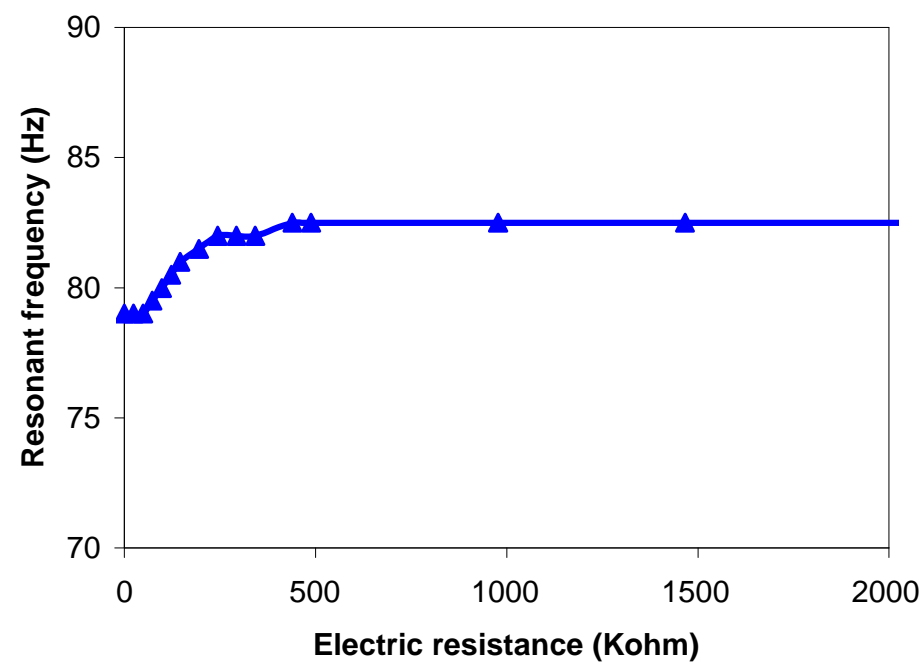

Figure 11 Resonant frequency shift verse the electric resistance connected to piezoelectric EHDs of piezoelectric layers in parallel

Table 1 Input geometric and material parameters used in the developed CPC-FEM

\begin{tabular}{|l|l|}
\hline \multicolumn{2}{|c|}{ Substrate material: brass } \\
\hline Young's modulus $(\mathrm{GPa})$ & 110 \\
\hline Poisson's ratio $\mu_{\mathrm{s}}$ & 0.23 \\
\hline Density $\left(\mathrm{kg} / \mathrm{m}^{3}\right)$ & 2330 \\
\hline $\begin{array}{l}\text { Length* } \times \text { width } \times \text { thickness }(\mathrm{mm}) \\
\text { * is the sum of length of mass and piezoelectric } \\
\text { layer }\end{array}$ & $28^{*} \times 3.2 \times 0.1016$ \\
\hline \multicolumn{2}{|l}{} \\
\hline
\end{tabular}




\begin{tabular}{|c|c|}
\hline \multicolumn{2}{|c|}{ Piezoelectric material: PSI-5H4E from Piezo Systems, Inc. } \\
\hline Young's modulus (GPa) & \\
\hline $\mathrm{E}_{11}$ & 62 \\
\hline $\mathrm{E}_{33}$ & 50 \\
\hline Poission's ratio & 0.3 \\
\hline Elastic constant:(GPa) & \\
\hline $\mathrm{C}_{11}$ & 110.8 \\
\hline $\mathrm{C}_{12}$ & 49.8 \\
\hline $\mathrm{C}_{13}$ & 49.8 \\
\hline $\mathrm{C}_{33}$ & 110.8 \\
\hline $\mathrm{C}_{44}$ & 30.5 \\
\hline Density $\rho_{\mathrm{p}}\left(\mathrm{kg} / \mathrm{m}^{3}\right)$ & 7800 \\
\hline $\begin{array}{c}\text { Piezoelectric constant }\left(\times 10^{-12} \mathrm{~m} / \text { volt }\right) \\
\mathrm{d}_{33} \\
\mathrm{~d}_{31}\end{array}$ & $\begin{array}{l}650 \\
-320\end{array}$ \\
\hline $\begin{array}{ll}\text { Coupling coefficient } & \\
& \mathrm{k}_{33} \\
\mathrm{k}_{31}\end{array}$ & $\begin{array}{l}0.75 \\
0.44\end{array}$ \\
\hline Relative dielectric constant $\quad \varepsilon_{33}$ & 3800 \\
\hline Mechanical Q & 32 \\
\hline Length $\times$ width $\times$ thickness $(\mathrm{mm})$ & $10.7 \times 3.2 \times 0.278$ \\
\hline \multicolumn{2}{|l|}{ Seismic mass: Tungsten } \\
\hline Young's modulus (GPa) & 400 \\
\hline Poisson ratio $\mu_{\mathrm{s}}$ & 0.28 \\
\hline Density $\left(\mathrm{kg} / \mathrm{m}^{3}\right)$ & 17000 \\
\hline Length $\times$ width $\times$ thickness $(\mathrm{mm})$ & $17.3 \times 3.6 \times 7.7$ \\
\hline
\end{tabular}

Table 2 Comparison of the outputs for configurations in parallel and series

\begin{tabular}{|c|c|c|c|}
\hline \multirow[t]{2}{*}{ Resistances } & \multirow[t]{2}{*}{ Outputs } & \multicolumn{2}{|c|}{ Piezoelectric layer configuration } \\
\hline & & In Parallel & In series \\
\hline \multirow[t]{4}{*}{$R=0$} & $I_{R-\max }(\mu \mathrm{A})$ & 140 & 70 \\
\hline & $V_{R-\max }($ Volt $)$ & 0 & 0 \\
\hline & $P_{R-\max }(\mu \mathrm{W})$ & 0 & 0 \\
\hline & $z_{\max }(\mu \mathrm{M})$ & 169 & 169 \\
\hline \multirow[t]{4}{*}{$R=\infty$} & $I_{R-\max }(\mu \mathrm{A})$ & 0 & 0 \\
\hline & $V_{R-\max }($ Volt $)$ & 15.77 & 31.6 \\
\hline & $P_{R-\max }(\mu \mathrm{W})$ & 0 & 0 \\
\hline & $z_{\max }(\mu \mathrm{M})$ & 178 & 178 \\
\hline \multirow{4}{*}{$R_{o p t}^{*}=\frac{1}{2 \pi f_{s c} C_{p}}$} & $I_{R-\max }(\mu \mathrm{A})$ & 22.3 & 11.2 \\
\hline & $V_{R-\max }($ Volt $)$ & 5.44 & 10.71 \\
\hline & $P_{R-\max }(\mu \mathrm{W})$ & 60.6 & 60.2 \\
\hline & $z_{\max }(\mu \mathrm{M})$ & 71 & 70.4 \\
\hline
\end{tabular}

